Revue internationale P.M.E.

Économie et gestion de la petite et moyenne entreprise

\title{
L'importance des liens faibles dans les entreprises artisanales : l'exemple d'Internet pour le partage et la réutilisation des connaissances
}

\section{Carole Marti}

Volume 19, numéro 3-4, 2006

URI : https://id.erudit.org/iderudit/1008504ar

DOI : https://doi.org/10.7202/1008504ar

Aller au sommaire du numéro

Éditeur(s)

Presses de l’Université du Québec

ISSN

0776-5436 (imprimé)

1918-9699 (numérique)

Découvrir la revue

Citer cet article

Marti, C. (2006). L'importance des liens faibles dans les entreprises artisanales: l'exemple d'Internet pour le partage et la réutilisation des connaissances. Revue internationale P.M.E., 19(3-4), 137-145. https://doi.org/10.7202/1008504ar
Résumé de l'article

Est-ce que Internet offre l'opportunité de construire de nouveaux réseaux à liens faibles ? Nous présentons dans cet article une recherche menée au sein d'une Chambre de métiers et de l'artisanat. Notre travail a consisté à mettre en place un portail interactif en ligne contenant des histoires sur l'utilisation des TIC par les artisans. Nous proposons une discussion du concept de liens faibles afin de comprendre leur importance pour partager et réutiliser des connaissances. 


\section{Notes de recherche}

\section{L'importance des liens faibles dans les entreprises artisanales: l'exemple d'Internet pour le partage et la réutilisation des connaissances}

Carole MARTI

CREGO, Université de Montpellier II

MOTS CLÉS

Artisans - Liens faibles - Partage - Réutilisation - Histoires

\section{L'AUTEURE}

CARole Martı est titulaire d'un doctorat en sciences de gestion à l'Université de Montpellier II. Elle est membre du Centre de recherche en gestion des organisations (CREGO - Montpellier EA 731). Ses travaux portent sur la gestion des connaissances et plus précisément sur les narrations comme outil de partage des expériences. Elle a publié ses travaux dans de nombreux congrès et dans deux ouvrages collectifs. Adresse : Université de Montpellier II, CREGO, place E.-Bataillon, 34095 Montpellier, France. Courriel : <marti@iae.univ-montp2.fr>.

\section{RÉSUMÉ}

Est-ce que Internet offre l'opportunité de construire de nouveaux réseaux à liens faibles? Nous présentons dans cet article une recherche menée au sein d'une Chambre de métiers et de l'artisanat. Notre travail a consisté à mettre en place un portail interactif en ligne contenant des histoires sur l'utilisation des TIC par les artisans. Nous proposons une discussion du concept de liens faibles afin de comprendre leur importance pour partager et réutiliser des connaissances. 


\begin{abstract}
Is there an opportunity with Internet to build new weak links networks for sharing knowledge and developing innovation? In this article, the research carried out in a French Regional Chamber of Trade and Crafts is described. Our work consisted in establishing an interactive portal collecting stories, about the experiences of craftsmen using ICT. We propose a discussion of the weak links concept, in order to understand the opportunity with Internet to build new weak links networks for sharing and reusing knowledge.
\end{abstract}

\title{
RESUMEN
}

¿Es que Internet ofrece la oportunidad de construir nuevas redes a vínculos escasas? Presentamos en este artículo una investigación llevada a cabo en una «Chambre de métiers et de l'artisanat». Nuestro trabajo consistió en establecer un pórtico interactivo en línea que contenía historias sobre la utilización del TIC por los artesanos. Proponemos un debate del concepto de vínculos escasos con el fin de que comprenda su importancia para compartir y reutilizar conocimientos.

\section{ZUSAMMENFASSUNG}

Bietet das Internet die Gelegenheit, neue lose Netzwerke von Beziehungen aufzubauen? Die in diesem Artikel dargestellte Forschung wurde in einer Handwerkskammer durchgeführt. Die Arbeit bestand darin, ein interaktives Portal aufzubauen mit Beispielen von Informationskommunikations-Technologien bei Handwerksbetrieben. Nach einer eingehenden Diskussion der losen Verbindungen, verstehen wir deren Wichtigkeit zur Verbreitung und zum Austausch von Wissen.

\section{Introduction}

De nombreuses études reconnaissent que la connaissance est la clé de la différenciation des petites entreprises mais aussi de leur compétitivité. Dans cet article, nous nous intéressons à la diffusion de ces connaissances par des réseaux à liens faibles qui s'opposent à ceux à liens forts. Granovetter (2000) définit les réseaux à liens faibles comme ceux avec lesquels les individus ont peu d'interactions dans le temps, une faible intensité émotionnelle, peu de confiance et peu de services réciproques. Les réseaux à liens faibles sont constitués par les sources moins fréquentées, à l'inverse des liens forts. Les liens forts sont sources de confiance et procurent une certaine sécurité, alors que les liens faibles donnent accès aux informations disponibles en dehors des cercles sociaux familiers (Granovetter, 1985). Les liens forts sont des liens interactifs (Leonard-Barton et Sinha,1993) et sont nécessaires pour l'échange de connaissances complexes, difficiles à codifier et souvent intégrées à un système de connaissances structurées (Hansen, 1999).

Revue internationale P.M.E., vol. 19, nos 3-4, 2006 
La capacité à innover semble d'autant plus importante que les idées se diffusent à travers des liens faibles (Ruef, 2002). Les relations sociales entre des individus différents favorisent l'adaptabilité à des situations nouvelles, puisque l'information sur de nouvelles opportunités y est plus facilement accessible. En ce qui concerne les PME, Julien, Andriambeloson et Ramangalahy (2002), à propos du comportement innovant de PME, concluent que les firmes les plus innovantes sont celles qui recourent le plus volontiers à ces liens faibles.

En effet, comparativement aux grandes entreprises, les petites entreprises (PE) n'ont pas les mêmes moyens, en termes de temps et de ressources financières, pour accéder à l'information. Aujourd'hui pourtant, le dirigeant de PE dispose lui aussi de sources informationnelles grâce aux technologies de l'information et de la communication (TIC). Et le moyen le plus «simple» de disposer d'informations pertinentes est devenu de passer par ces nouveaux réseaux. Dans cette note de recherche, nous allons tenter de répondre à la question suivante:

Quelles sont les opportunités qu'offre Internet dans la construction de ces nouveaux réseaux à liens faibles pour le partage et la réutilisation des connaissances dans les petites entreprises?

Pour y répondre, nous verrons dans la première partie la création des liens faibles grâce aux TIC. Dans une seconde, nous illustrerons nos propos par une étude des opportunités liées aux réseaux à liens faibles «sur Internet ». Les artisans peuvent aujourd'hui y partager et réutiliser des connaissances.

\section{La création de liens faibles par les TIC}

Par le biais de l'analyse de réseaux sociaux, Granovetter (1973) montre que les interactions interindividuelles se traduisent par des phénomènes collectifs qui rétroagissent, de manière symétrique, sur les individus et les petits groupes. Au cœur de son analyse se trouve la notion de «lien interpersonnel». Granovetter (2000) le définit comme un «lien dont la force est une combinaison (probablement linaire) de la quantité de temps, de l'intensité émotionnelle, de l'intimité (la confiance mutuelle) et des services réciproques qui caractérisent ce lien ».

En fonction de l'intensité de la relation, le lien est qualifié de fort, faible ou absent et son intensité modifie la propension des agents à innover (Ruef, 2002). Granovetter (2000) trouve un résultat contre-intuitif selon lequel l'efficacité informationnelle, c'est-à-dire la vitesse et la richesse de l'information diffusée dans l'ensemble d'un réseau est en fait supérieure dans les réseaux 
de liens faibles, car «les individus avec qui on est faiblement lié ont plus de chances d'évoluer dans des cercles différents et ils ont donc accès à des informations différentes de celles que l'on reçoit»(Granovetter 2000).

Autrement dit, l'existence de liens faibles entre agents, c'est-à-dire de relations relativement peu fréquentes, est déterminante dans la dynamique de circulation de l'information dans les réseaux sociaux.

Cette problématique est prégnante dans le contexte de la PE. Comme le soulignent Julien, Andriambeloson et Ramangalahy (2002), compte tenu de leurs ressources limitées, les PME doivent recourir à différents réseaux afin d'obtenir une information riche. Le rôle de ces réseaux est d'aider ce type d'entreprises à profiter de sources informationnelles diverses. Les auteurs rappellent que, dans les PME, les réseaux sont essentiellement des réseaux personnels $^{1}$ et d'affaires ${ }^{2}$ qui sont le plus souvent des réseaux forts. Mais certains réseaux, plus purement informationnels, qui peuvent comprendre aussi bien des réseaux sociaux qu'institutionnels, peuvent être à signaux forts ou faibles. Ces réseaux informationnels servent alors à compléter les informations utiles provenant des réseaux personnels et d'affaires.

Comme dans une petite entreprise, le rôle du dirigeant est déterminant sinon quasi exclusif dans ce processus, il est justement très important qu'il puisse s'approprier les outils qui lui permettront de mieux jouer son rôle d'interface: l'avantage concurrentiel peut alors venir, non pas de la créativité d'un seul individu, mais de la capacité à construire un réseau relationnel innovant «à liens faibles » (Julien, Andriambeloson et Ramangalahy, 2002). Par opposition aux traditionnels réseaux à liens forts (que constitue, par exemple, l'expert-comptable, le banquier, les fournisseurs, les clients, la Chambre de métiers, etc.), ces réseaux à liens faibles sont sociologiquement plus éloignés du dirigeant. Néanmoins, ils peuvent favoriser le repérage des signaux faibles que préconisent Janissek-Muniz et Lesca (2003), afin d'aider l'ensemble de l'entreprise à apprendre de façon permanente. Pour les artisans, de nombreux moyens existent pour construire ces réseaux à liens faibles (les stages de formation, les clubs de dirigeants, les salons professionnels, la veille sur Internet, l'accueil de stagiaires, les expositions, la formation en ligne, le parrainage de seniors, etc.).

1. Ce sont les réseaux particuliers à chaque entrepreneur; ils comprennent généralement un ou deux amis, quelques membres clés du personnel, un collègue d'études, un membre du club d'entrepreneurs (Julien, Andriambeloson et Ramangalahy, 2002).

2. Ils englobent les entreprises avec lesquelles l'entreprise fait couramment des affaires (Julien, Andriambeloson et Ramangalahy, 2002). 
Internet peut permettre alors de décupler les capacités des entreprises, pour autant que l'on sache en profiter. Pour Granovetter (1973), ce sont bien les réseaux dans lesquels les acteurs sont encastrés qui vont déterminer les comportements individuels et le rendement de leurs échanges économiques et Internet permettrait, par exemple, à presque toutes les petites entreprises de tisser des liens à l'international.

Cochoy (2003) a coordonné un numéro de la revue Réseaux entièrement consacré aux TIC dans le cadre des petites entreprises et il arrive à la conclusion

[...] qu'au travers des récits recueillis, on constate la réalisation de petites expériences et l'engagement d'apprentissages progressifs; on observe chez les acteurs l'affirmation d'une remarquable capacité d'appropriation des outils et l'avènement d'une étonnante conscience réflexive de leurs potentialités. Internet permet aux petits acteurs, pour la première fois peut-être, d'être visibles sur le marché international sans devoir assumer des coûts de publicité hors de portée pour eux; Internet leur donne l'occasion de mobiliser gratuitement des informations sur le marché international sans avoir à se découvrir; Internet leur fournit enfin les moyens de préserver d'anciennes relations et/ou de nouer de nouveaux contacts (Cochoy, 2003).

Ces nouveaux points de vue concernant la capacité d'innovation des petites entreprises sont dus en partie aux progrès réalisés dans son évaluation $^{3}$ et surtout au fait que l'environnement économique et social a changé avec les évolutions technologiques, l'obsolescence et la mondialisation: nous serions dans une ère de jeunes entreprises (Jovanovic, 2001). De ce fait, un certain nombre d'explications théoriques ont émergé pour expliquer les avantages des petites entreprises en matière d'innovation dans certains secteurs : l'absence de contraintes bureaucratiques, les nombreuses «petites » innovations trop modestes pour intéresser les grandes entreprises, les stratégies de saisie des opportunités, les innovations souvent commerciales et incrémentales, des proximités professionnelles et/ou communautaires, les externalités informationnelles au sein des réseaux, les systèmes productifs locaux, l'enthousiasme possible dans la petite entreprise où les liens entre les défis, le personnel et les récompenses potentielles sont étroits.

L'analyse de la littérature sur les réseaux à liens faibles est une approche intéressante pour la PE. Les TIC et particulièrement Internet

3. Dans les secteurs très innovants, où les petites entreprises ne représentent pas une part d'emplois très élevée, on peut montrer que l'avantage innovant relatif est détenu par les petites entreprises (Commisson européenne, 2003). 
permettent de développer des réseaux à liens faibles et constituent des sources informationnelles supplémentaires pour obtenir, partager et réutiliser des informations.

\section{Expérimentation: le partage et la réutilisation des connaissances grâce à Internet}

Nous allons illustrer nos propos par un travail que nous avons mené au sein de la Chambre régionale de métiers et de l'artisanat (CRMA) du LanguedocRoussillon (Marti, 2005).

Les TIC constituent un axe de travail transversal en plein essor au sein des Chambres de métiers ${ }^{4}$ et la CRMA a souhaité mener un projet d'action en faveur du développement de la compétitivité des artisans. Elle justifie ce choix par l'analyse du contexte et part de l'idée que l'utilisation de ces technologies est un facteur de développement et de gains de compétitivité, sous réserve que cette utilisation soit maîtrisée et cohérente avec le métier de l'entreprise: le secteur est particulièrement réceptif à l'innovation, du fait du pragmatisme des artisans, de leur culture technique et de la capacité d'adaptation des petites entreprises. Toutefois, comme le soulignent Carrier et Garand (1996), la majorité des innovations observées sont des innovations de routine (réutilisation avec adaptation) s'inscrivant à l'intérieur d'une démarche incrémentale plutôt qu'à des innovations radicales.

L'outil construit dans cette étude doit répondre à ce besoin latent, il regroupe «en ligne» un certain nombre d'histoires racontées par des artisans à propos de leurs usages des TIC. Notre méthodologie d'accès au terrain a été celle d'une recherche-action. Elle se justifie par le fait que nous avons mené de concert l'action et la recherche afin d'améliorer une situation problématique. Ici, il s'agit de permettre la construction d'un portail permettant le recueil d'expériences d'artisans avec les TIC afin de les rendre accessibles à d'autres artisans. Nous avons choisi les histoires pour rendre compte de ces expériences. Cette forme de diffusion des connaissances permet d'améliorer la capacité d'apprentissage des individus grâce aux propriétés des récits pour collecter, structurer et diffuser les expériences (Marti, 2006). Une fois l'outil en ligne, nous avons mené une expérimentation par l'étude de 48 cas d'entreprises artisanales. Notre protocole d'expérimentation s'est déroulé en trois étapes: avant, pendant et après la navigation sur le site.

4. Le rôle d'une CRMA est de réaliser des études prospectives afin d'orienter le développement à moyen terme du secteur de l'artisanat et du fonctionnement des Chambres régionales. 
Le principal objectif de cette expérimentation est de montrer le partage et la réutilisation de connaissances. Par ailleurs, nous avons mis en évidence que l'artisan désireux de partager des connaissances dispose de différents moyens: d'une part, des réseaux à liens forts (par exemple, l'expertcomptable) et, d'autre part, des réseaux à liens faibles (par exemple, les salons professionnels). L'artisan possède un large éventail de sources de connaissances en fonction des questions qu'il se pose. Aujourd'hui, cet outil de gestion de connaissances fournit une nouvelle source de connaissances: le protocole d'expérimentation a permis non seulement de vérifier qu'il y a bien création de liens faibles entres des artisans qui ne se rencontrent pas en face à face, mais qu'ils peuvent profiter des connaissances développées par d'autres.

Sur les 48 cas analysés, nous avons pu constater que tous ne réutilisent pas de la même façon: certains vont plutôt réutiliser des histoires proches de leur contexte professionnel, alors que d'autres, au contraire, vont réutiliser des histoires éloignées de leur contexte. En effet, l'analyse des 48 cas a permis le repérage de trois types de scénarios de réutilisation. Nous trouvons 12 artisans dans un premier scénario «j'adopte directement à l'intérieur »; ici, la personne envisage une réutilisation lorsqu'elle se sent proche du contexte de l'histoire qu'elle lit. L'ensemble des artisans retient une connaissance très contextualisée, gère difficilement la distance cognitive ${ }^{5}$; lorsqu'ils envisagent de réutiliser, ils le font sans faire de modification. Dans une deuxième catégorie, nous avons 15 artisans dans le scénario «j'adapte à l'intérieur et à l'extérieur»; nous remarquons qu'ils gèrent facilement la distance cognitive. Quand ils envisagent de réutiliser, ils adaptent la connaissance à leur contexte professionnel. Nous recensons 11 cas dans un troisième groupe: le scénario «je transforme à l'intérieur et à l'extérieur»; ici, les artisans gèrent facilement la distance cognitive, la lecture d'histoires leur permettant de trouver de nouvelles idées. Enfin, 10 cas n'appartiennent à aucun de ces scénarios: ils relèvent de la catégorie des non-réutilisateurs.

L'activation de liens faibles par Internet afin de partager et de réutiliser des connaissances s'est révélée fructueuse. Nous concluons que les narrations ont permis aux artisans de partager et de réutiliser des connaissances.

5. La distance ou la proximité cognitive de deux individus correspond au nombre de connaissances qu'ils ont en commun. Plus deux individus sont proches cognitivement, plus ils ont des connaissances en commun. 


\section{Conclusion}

Les relations entre des individus de réseaux sociaux différents favorisent l'adaptabilité à des situations nouvelles. La capacité à innover semble d'autant plus importante que les idées se diffusent à travers des liens faibles. De nombreuses études ont montré que les firmes les plus innovantes sont celles qui recourent le plus à ces liens faibles. La présence au sein d'un ensemble social d'une alternance de liens forts et faibles induit les «trous structuraux» nécessaires à de nouvelles relations sociales et à de nouvelles opportunités.

Nous avons montré que le recours aux TIC permet de développer des réseaux à liens faibles et qu'ils deviennent une nouvelle source informationnelle. Par ailleurs, notre recherche met en évidence trois scénarios de partage puis de réutilisation de connaissances par l'activation de ces liens.

\section{Bibliographie}

CARrier, C. et D. Garand (1996), «Le concept d'innovation: débats et ambiguïtés », $\mathrm{V}^{\mathrm{e}}$ Conférence internationale de management stratégique (AIMS), Lille.

Cochoy, F. (2003), «La «toile» comme force des points faibles?», Réseaux, $\mathrm{n}^{\circ} 121$, p. $119-144$.

Commission européenne (2003), Observatoire des PME européennes, Bruxelles.

Granovetter, M. (1973), «The strength of weak ties», American Journal of Sociology, vol. $78, \mathrm{n}^{\circ}$ 6, p. 1360-1380.

Granovetter, M. (1985), «Economic action and social structure: the problem of embeddedness », American Journal of Sociology, vol. 78, n 6, p. 481-510.

Granovetter, M. (2000), Le marché autrement: les réseaux dans l'économie, Paris, Desclée de Brouwer.

HANSEN, M.T. (1999), «The search-transfer problem: the role of weak ties in sharing knowledge across organization subunits», Administrative Science Quarterly, vol. $44, \mathrm{n}^{\circ} 1$, p. $82-111$.

JANissek-Muniz, R. et H. Lesca (2003), «Veille stratégique: application d'Internet et sites Web pour "provoquer" des informations à caractère anticipatif», Association Information et Management (AIM), Grenoble.

Jovanovic, B. (2001), «New technology and the small firm», Small Business Economics, vol. $16, \mathrm{n}^{\circ} 1$, p. $53-55$.

Julien, P.-A., E. Andriambeloson et C. Ramangalahy (2002), «Réseaux, signaux faibles et innovation technologique dans les PME du secteur des équipements de transport terrestre», VI ${ }^{\mathrm{e}}$ Congrès international francophone de la PME, HEC Montréal. 
Leonard-Barton, D. et D.K. Sinha (1993), «Developer-user interaction and user satisfaction in internal technology transfer », Academy of Management Journal, vol. $36, \mathrm{n}^{\circ}$ 5, p. 1125-1139.

MARTI, C. (2005), L'apport des méthodes narratives à la gestion des connaissances: le partage et la réutilisation entre artisans, Thèse de doctorat en sciences de gestion, Université de Montpellier II, 398 p.

Marti, C. (2006), «Des histoires... à la gestion des connaissances», dans E. Soulier (dir.), Le storytelling: concepts, outils et applications, Paris, Éditions Hermes Science.

RuEF, M. (2002), «Strong ties, weak ties and islands: structural and cultural predictors of organizational», Industrial and Corporate Change, vol. 11, n 3, p. 427-449.

Revue internationale P.M.E., vol. 19, $\mathrm{n}^{\text {os }}$ 3-4, 2006

(C) 2006 - Presses de l'Université du Québec

Édifice Le Delta I, 2875, boul. Laurier, bureau 450, Québec, Québec G1V 2M2 • Tél.: (418) 657-4399 - www.puq.ca 